\title{
Nanocrystalline Diamond Grids for FIB Specimen Preparation and S/TEM Analytics
}

Lucille Giannuzzi $^{1}$, Nicolaie Moldovan ${ }^{2}$, Jamie Trindell ${ }^{3}$ and Joshua Sugar ${ }^{4}$

${ }^{1}$ EXpressLO LLC, Lehigh Acres, Florida, United States, ${ }^{2}$ Alcorix Co., Plainfield, Illinois, United States,

${ }^{3}$ Sandia National Lab, Pleasanton, California, United States, ${ }^{4}$ Sandia National Laboratories, Livermore, California, United States

Focused ion beam (FIB) specimen half grids for scanning/transmission electron microscopy (S/TEM) are generally available in $\mathrm{Cu}$, Mo, or $\mathrm{Ni}$. However, these metal grids can generate spurious X-ray fluorescence and complicate analytical analyses, particularly if the specimen contains one of these grid elements. Thus, we have developed FIB grids for S/TEM using a low atomic number (Z) material to reduce fluorescence of grid material for accurate analytical work.

Nanocrystalline diamond (NCD) grids have been produced using conventional lithography and chemical vapor deposition methods having slots and dimensions consistent with previously developed grids for FIB ex situ lift out (EXLO) [1]. While not developed for in situ lift out (INLO) per se, they can indeed be used for FIB INLO techniques. The advantages to the low-Z NCD grids are that they are conductive, can be subjected to high temperature heating experiments, and are excellent for analytical work since X-ray fluorescence or high energy overlaps are reduced or completely eliminated. The $3 \mathrm{~mm}$ NCD grids were produced in either a half or full $3 \mathrm{~mm}$ grid geometry, allowing use for either FIB EXLO or INLO specimen preparation, or for thin film analyses [2].

Figure 1 shows images of the 3mm NCD half grids and full grids. Figure 2a shows $30 \mathrm{keV}$ SEM energy dispersive spectrometry (EDS) data from the NCD grid surface. Note that $\mathrm{C} \mathrm{Ka}$ is the only X-ray peak detected from these NCD grids. Shown in figures $2 b, c$ is a comparison of EDS spectra obtained using $200 \mathrm{keV}$ STEM from a Ga FIB prepared $(\mathrm{Ca}, \mathrm{Ce})(\mathrm{Mn}, \mathrm{Ti}) \mathrm{O}_{3}$ specimen mounted on a Mo half grid versus an NCD half grid. Note the presence of all the metal specimen constituents in the low energy X-ray regime in figure $2 \mathrm{~b}$. The high energy X-ray regime in figure $2 \mathrm{c}$ shows a small amount of Ga expected from the FIB milling, but also shows fluorescence due to apparent system peaks from possibly the STEM holder $(\mathrm{Cu})$, grid $(\mathrm{Mo})$, and EDS collimator $(\mathrm{Pb}, \mathrm{Zr}, \mathrm{Mo})$. Note that the intensity of all the system peaks are reduced when the NCD grid is used. Note also that the Mo Ka and Mo Kb peaks are reduced significantly and the Mo La is eliminatedwhen the NCD grid is used.
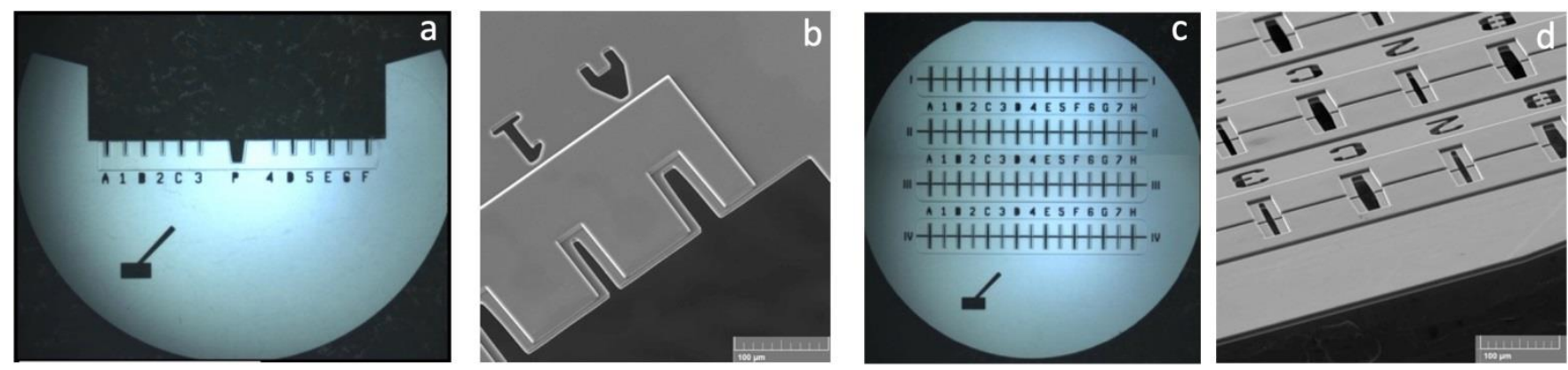

Figure 1. (a) NCD half grid, (b) slot region of the NCD half grid, (c) NCD full grid, and (d) slot region of the NCD full grid. 

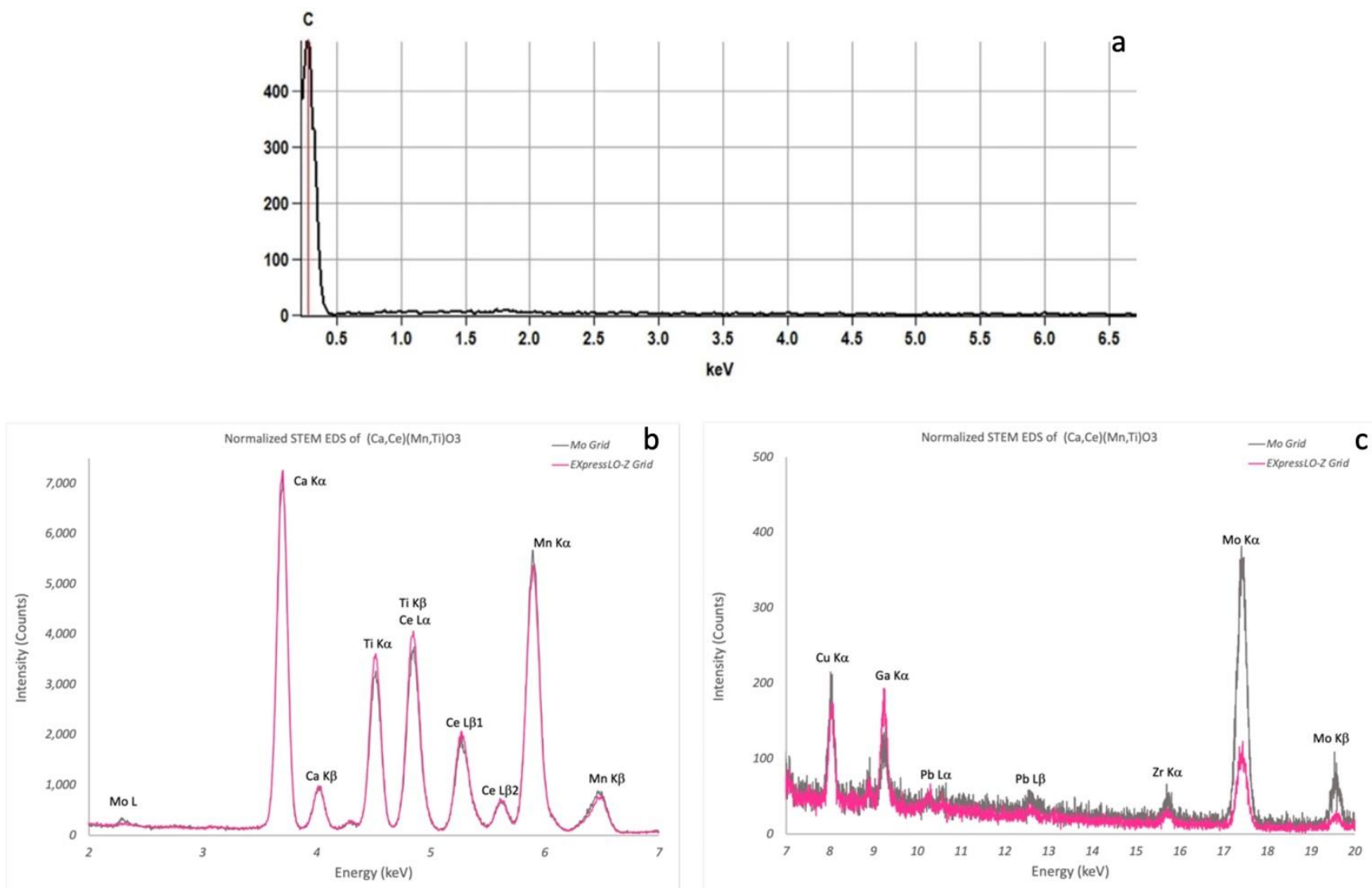

Figure 2. (a) $30 \mathrm{keV}$ SEM EDS of the NCD grid and (b,c) comparison of $200 \mathrm{keV}$ STEM EDS of a Ga FIB prepared $(\mathrm{Ca}, \mathrm{Ce})(\mathrm{Mn}, \mathrm{Ti}) \mathrm{O} 3$ specimen on Mo half grid vs. NCD half grid.

\section{References}

[1] L.A. Giannuzzi et al., Microsc. Microanal., 21 (2015) p. 1034.

[2] The NCD grid design and methods for EXLO are covered under US Patents 8,740,209; 8,789,826; $10,522,324 ; 10,801,926$. 